\title{
A MULTIPLICITY RESULT FOR DOUBLE SINGULARLY PERTURBED ELLIPTIC SYSTEMS
}

\author{
MARCO GHIMENTI AND ANNA MARIA MICHELETTI
}

\begin{abstract}
We show that the number of low energy solutions of a double singularly perturbed Schroedinger Maxwell system type on a smooth 3 dimensional manifold $(M, g)$ depends on the topological properties of the manifold.

The result is obtained via Lusternik Schnirelmann category theory.
\end{abstract}

\section{INTRODUCTION}

Given real numbers $q>0, \omega>0, p>4$, we consider the following system of Schroedinger Maxwell type on a smooth manifold $M$ endowed with a Riemannian metric $g$

$$
\left\{\begin{array}{cc}
-\varepsilon^{2} \Delta_{g} u+u+\omega u v=|u|^{p-2} u & \text { in } M \\
-\varepsilon^{2} \Delta_{g} v+v=q u^{2} & \text { in } M \\
u>0 \text { in } M &
\end{array}\right.
$$

where $\Delta_{g}$ is the Laplace-Beltrami operator on $M$.

We want to prove that when the parameter $\varepsilon$ is sufficiently small, there are many low energy solution of (1). In particular the number of solutions of (1) is related to the topology of the manifold $M$. We suppose without loss of generality, that the manifold $M$ is isometrically embedded in $\mathbb{R}^{n}$ for some $n$.

Here there is a competition between the two equation, since both share the same singular perturbation of order $\varepsilon^{2}$. In [10, 11] we dealt with a similar system where only the first equation had a singular perturbation. In this case the second equation disappears in the limit. In Section 2.1 we write the limit problem taking care of the competition, and we find the model solution for system (1).

A problem similar to (1), namely the Schroedinger-Newton system, has been studied from a dynamical point of view in [9]. Also in this paper the two equation have the $\varepsilon^{2}$ singular perturbation.

Recently, Schroedinger Maxwell type systems received considerable attention from the mathematical community, we refer, e.g. to [1, 2, 5, 6, 7, 8, 15, 16]. A special case of Schroedinger Maxwell type systems, namely when the system is set in $\mathbb{R}^{3}$, takes the name of Schroedinger-Poisson-Slater equation and it arises in Slater approximation of the Hartree-Fock model. We want here to especially mention some result of the existence of solutions, i.e. [2, 7, 12, 15, 17, since the limit problem (1) is a Schroedinger-Poisson-Slater type equation. (for a more exhaustive discussion on

2010 Mathematics Subject Classification. 35J60,35J47,58E05,81V10.

Key words and phrases. Riemannian manifold, Schroedinger Maxwell systems, Schroedinger Poisson Slater equation, singular pertubation.

The first author was supported by Gruppo Nazionale per l'Analisi Matematica, la Probabilità e le loro Applicazioni (GNAMPA) of Istituto Nazionale di Alta Matematica (INdAM). 
Schroedinger-Poisson-Slater and on the physical models that leads to this equation we we refer to [13, 14] and the references therein).

Our main results is the following.

Theorem 1. Let $4<p<6$. For $\varepsilon$ small enough there exist at least cat $(M)$ positive solutions of (11).

Here we recall the definition of the Lusternik Schnirelmann category of a set.

Definition 2. Let $X$ a topological space and consider a closed subset $A \subset X$. We say that $A$ has category $k$ relative to $X$ ( $\operatorname{cat}_{X} A=k$ ) if $A$ is covered by $k$ closed sets $A_{j}, j=1, \ldots, k$, which are contractible in $X$, and $k$ is the minimum integer with this property. We simply denote cat $X=\operatorname{cat}_{X} X$.

\section{Preliminary Results}

We endow $H^{1}(M)$ and $L^{p}(M)$ with the following equivalent norm

$$
\begin{array}{cc}
\|u\|_{\varepsilon}^{2}=\frac{1}{\varepsilon^{3}} \int_{M} \varepsilon^{2}|\nabla u|^{2}+u^{2} d \mu_{g} & |u|_{\varepsilon, p}^{p}=\frac{1}{\varepsilon^{3}} \int_{M}|u|^{p} d \mu_{g} \\
\|u\|_{H^{1}}^{2}=\int_{M}|\nabla u|^{2}+v^{2} d \mu_{g} & |u|_{p}^{p}=\int_{M}|u|^{p} d \mu_{g}
\end{array}
$$

and we refer to $H_{\varepsilon}$ (resp. $\left.L_{\varepsilon}^{p}\right)$ as space $H^{1}(M)$ (resp. $L_{\varepsilon}^{p}$ ) endowed with the $\|\cdot\|_{\varepsilon}$ (resp. $\left.|\cdot|_{\varepsilon, p}\right)$ norm. Obviously, we refer to the scalar product on $H_{\varepsilon}$ as

$$
\langle u, v\rangle_{\varepsilon}=\frac{1}{\varepsilon^{3}} \int_{M} \varepsilon^{2} \nabla u \nabla v+u v d \mu_{g} .
$$

Following an idea by Benci and Fortunato [5], for any $\varepsilon$ we introduce the map $\psi_{\varepsilon}: H^{1}(M) \rightarrow H^{1}(M)$ that is the solution of the equation

$$
-\varepsilon^{2} \Delta_{g} v+v=q u^{2} \text { in } M
$$

Lemma 3. The map $\psi: H^{1}(M) \rightarrow H^{1}(M)$ is of class $C^{2}$ with derivatives $\psi^{\prime}(u)$ and $\psi^{\prime \prime}(u)$ which satisfy

$$
\begin{aligned}
-\varepsilon^{2} \Delta_{g} \psi_{\varepsilon}^{\prime}(u)[\varphi]+\psi_{\varepsilon}^{\prime}(u)[\varphi] & =2 q u \varphi \\
-\varepsilon^{2} \Delta_{g} \psi_{\varepsilon}^{\prime \prime}(u)\left[\varphi_{1}, \varphi_{2}\right]+\psi_{\varepsilon}^{\prime \prime}(u)\left[\varphi_{1}, \varphi_{2}\right] & =2 q \varphi_{1} \varphi_{2}
\end{aligned}
$$

for any $\varphi, \varphi_{1}, \varphi_{2} \in H^{1}(M)$. Moreover $\psi_{\varepsilon}(u) \geq 0$.

Proof. The proof is standard.

Remark 4. We observe that by simple computation, for any $t>0$ we have that $\psi_{\varepsilon}(t u)=t^{2} \psi_{\varepsilon}(u)$. In fact, if $\psi_{\varepsilon}(u)$ solves (2), multiplying by $t^{2}$ both sides of (2) we get the claim.

Lemma 5. The map $T_{\varepsilon}: H_{\varepsilon} \rightarrow \mathbb{R}$ given by

$$
T_{\varepsilon}(u)=\int_{M} u^{2} \psi_{\varepsilon}(u) d \mu_{g}
$$

is a $C^{2}$ map and its first derivative is

$$
T_{\varepsilon}^{\prime}(u)[\varphi]=4 \int_{M} \varphi u \psi_{\varepsilon}(u) d \mu_{g} .
$$


Proof. The regularity is standard. The first derivative is

$$
T_{\varepsilon}^{\prime}(u)[\varphi]=2 \int u \varphi \psi_{\varepsilon}(u)+\int u^{2} \psi_{\varepsilon}^{\prime}(u)[\varphi] .
$$

By (3) and (2) we have

$$
\begin{aligned}
2 \int u \varphi \psi_{\varepsilon}(u) & =\frac{1}{q}\left(-\varepsilon^{2} \int \Delta\left(\psi_{\varepsilon}^{\prime}(u)[\varphi]\right) \psi_{\varepsilon}(u)+\int \psi_{\varepsilon}^{\prime}(u)[\varphi] \psi_{\varepsilon}(u)\right) \\
& =\frac{1}{q}\left(-\varepsilon^{2} \int \psi_{\varepsilon}^{\prime}(u)[\varphi] \Delta \psi_{\varepsilon}(u)+\int \psi_{\varepsilon}^{\prime}(u)[\varphi] \psi_{\varepsilon}(u)\right) \\
& =\int \psi_{\varepsilon}^{\prime}(u)[\varphi] u^{2}
\end{aligned}
$$

and the claim follows.

At this point we consider the following functional $I_{\varepsilon} \in C^{2}\left(H_{\varepsilon}, \mathbb{R}\right)$.

$$
I_{\varepsilon}(u)=\frac{1}{2}\|u\|_{\varepsilon}^{2}+\frac{\omega}{4} G_{\varepsilon}(u)-\frac{1}{p}\left|u^{+}\right|_{\varepsilon, p}^{p}
$$

where

$$
G_{\varepsilon}(u)=\frac{1}{\varepsilon^{3}} \int_{\Omega} u^{2} \psi_{\varepsilon}(u) d x=\frac{1}{\varepsilon^{3}} T_{\varepsilon}(u) .
$$

By Lemma 5 we have

$$
\begin{gathered}
I_{\varepsilon}^{\prime}(u)[\varphi]=\frac{1}{\varepsilon^{3}} \int_{\Omega} \varepsilon^{2} \nabla u \nabla \varphi+u \varphi+\omega u \psi_{\varepsilon}(u) \varphi-\left(u^{+}\right)^{p-1} \varphi \\
I_{\varepsilon}^{\prime}(u)[u]=\|u\|_{\varepsilon}^{2}+\omega G_{\varepsilon}(u)-\left|u^{+}\right|_{\varepsilon, p}^{p}
\end{gathered}
$$

then if $u$ is a critical points of the functional $I_{\varepsilon}$ the pair of positive functions $\left(u, \psi_{\varepsilon}(u)\right)$ is a solution of (11).

We define the following Nehari set

$$
\mathcal{N}_{\varepsilon}=\left\{u \in H^{1}(M) \backslash 0: N_{\varepsilon}(u):=I_{\varepsilon}^{\prime}(u)[u]=0\right\}
$$

The Nehari set has the following properties (for a complete proof see [10])

Lemma 6. If $p>4, \mathcal{N}_{\varepsilon}$ is a $C^{2}$ manifold and $\inf _{\mathcal{N}_{\varepsilon}}\|u\|_{\varepsilon}>0$.

If $u \in \mathcal{N}_{\varepsilon}$, then

$$
\begin{aligned}
I_{\varepsilon}(u) & =\left(\frac{1}{2}-\frac{1}{p}\right)\|u\|_{\varepsilon}^{2}+\omega\left(\frac{1}{4}-\frac{1}{p}\right) G_{\varepsilon}(u) \\
& =\left(\frac{1}{2}-\frac{1}{p}\right)\left|u^{+}\right|_{p, \varepsilon}^{p}-\frac{\omega}{4} G_{\varepsilon}(u) \\
& =\frac{1}{4}\|u\|_{\varepsilon}^{2}+\left(\frac{1}{4}-\frac{1}{p}\right)\left|u^{+}\right|_{p, \varepsilon}^{p} .
\end{aligned}
$$

and it holds Palais-Smale condition for the functional $I_{\varepsilon}$ on $\mathcal{N}_{\varepsilon}$.

Finally, for all $w \in H^{1}(M)$ such that $\left|w^{+}\right|_{\varepsilon, p}=1$ there exists a unique positive number $t_{\varepsilon}=t_{\varepsilon}(w)$ such that $t_{\varepsilon}(w) w \in \mathcal{N}_{\varepsilon}$. The number $t_{\varepsilon}$ is the critical point of the function

$$
H(t)=I_{\varepsilon}(t w)=\frac{1}{2} t^{2}\|w\|_{\varepsilon}^{2}+\frac{t^{4}}{4} \omega G_{\varepsilon}(w)-\frac{t^{p}}{p} .
$$


2.1. The limit problem. Consider the following problem in the whole space.

$$
\left\{\begin{array}{cc}
-\Delta u+u+\omega u v=|u|^{p-2} u & \text { in } \mathbb{R}^{3} \\
-\Delta v+v=q u^{2} & \text { in } \mathbb{R}^{3} \\
u>0 \text { in } \mathbb{R}^{3} &
\end{array}\right.
$$

In an analogous way we define the function $\psi_{\infty}(u)$ as a solution of the second equation and, as before, we can define a functional

$$
I_{\infty}(u)=\frac{1}{2}\|u\|_{H^{1}}^{2}+\frac{\omega}{4} G(u)-\frac{1}{p}\left|u^{+}\right|_{p}^{p}
$$

where $G(u)=\int_{\mathbb{R}^{3}} u^{2} \psi_{\infty}(u) d x$ and the Nehari manifold $\mathcal{N}_{\infty}=\left\{u \in H^{1}\left(\mathbb{R}^{3}\right) \backslash 0: I_{\infty}^{\prime}(u)[u]=0\right\}$. It is easy to prove (see [12]) that the value

$$
m_{\infty}=\inf _{\mathcal{N}_{\infty}} I_{\infty}
$$

is attained at least by a function $U$ which is a solution of problem (7).

We will refer at problem (7) as the limit problem. We set

$$
U_{\varepsilon}(x)=U\left(\frac{x}{\varepsilon}\right)
$$

and the function $U_{\varepsilon}$ will be the model solution for a solution of problem (11).

\section{MAIn InGREDIEnT OF THE PROOF}

We sketch the proof of Theorem 1. First of all, it is easy to see that the functional $I_{\varepsilon} \in C^{2}$ is bounded below and satisfies Palais Smale condition on the complete $C^{2}$ manifold $\mathcal{N}_{\varepsilon}$. Then we have, by well known results, that $I_{\varepsilon}$ has at least cat $I_{\varepsilon}^{d}$ critical points in the sublevel

$$
I_{\varepsilon}^{d}=\left\{u \in H^{1}: I_{\varepsilon}(u) \leq d\right\} .
$$

We prove that, for $\varepsilon$ and $\delta$ small enough, it holds

$$
\text { cat } M \leq \operatorname{cat}\left(\mathcal{N}_{\varepsilon} \cap I_{\varepsilon}^{m_{\infty}+\delta}\right)
$$

where $m_{\infty}$ has been defined in the previous section.

To get the inequality cat $M \leq$ cat $\left(\mathcal{N}_{\varepsilon} \cap I_{\varepsilon}^{m_{\infty}+\delta}\right)$ we build two continuous operators

$$
\begin{aligned}
\Phi_{\varepsilon} & : \quad M \rightarrow \mathcal{N}_{\varepsilon} \cap I_{\varepsilon}^{m_{\infty}+\delta} \\
\beta & : \quad \mathcal{N}_{\varepsilon} \cap I_{\varepsilon}^{m_{\infty}+\delta} \rightarrow M^{+} .
\end{aligned}
$$

where

$$
M^{+}=\left\{x \in \mathbb{R}^{n}: d(x, M)<R\right\}
$$

with $R$ small enough so that $\operatorname{cat}\left(M^{+}\right)=\operatorname{cat}(M)$. Without loss of generality, we can suppose $R=r$ the injectivity radius of $M$, in order to simplify the notations.

Following an idea in [4], we build these operators $\Phi_{\varepsilon}$ and $\beta$ such that $\beta \circ \Phi_{\varepsilon}$ : $M \rightarrow M^{+}$is homotopic to the immersion $i: M \rightarrow M^{+}$. By a classical result on topology (which we summarize in Remark 7) we have

$$
\operatorname{cat} M \leq \operatorname{cat}\left(\mathcal{N}_{\varepsilon} \cap I_{\varepsilon}^{m_{\infty}+\delta}\right)
$$

and the first claim of Theorem 1 is proved. 
Remark 7. Let $X_{1}$ and $X_{2}, X_{3}$ be topological spaces with $X_{1}$ and $X_{3}$ which are homotopically identical. If $g_{1}: X_{1} \rightarrow X_{2}$ and $g_{2}: X_{2} \rightarrow X_{3}$ are continuous operators such that $g_{2} \circ g_{1}$ is homotopic to the identity on $X_{1}$, then cat $X_{1} \leq$ cat $X_{2}$

\section{The MAP $\Phi_{\varepsilon}$}

For every $\xi \in M$ we define the function

$$
W_{\xi, \varepsilon}(x)=U_{\varepsilon}\left(\exp _{\xi}^{-1} x\right) \chi\left(\left|\exp _{\xi}^{-1} x\right|\right)
$$

where $\chi: \mathbb{R}^{+} \rightarrow \mathbb{R}^{+}$is a cut off function, that is $\chi \equiv 1$ for $t \in[0, r / 2), \chi \equiv 0$ for $t>r$ and $\left|\chi^{\prime}(t)\right| \leq 2 / r$. Here $\exp _{\xi}$ are the normal coordinates centered in $\xi \in M$ and $r$ is the injectivity radius of $M$. We recall the following well known expansion of the metric $g$ in normal coordinates:

$$
g_{i j}(\varepsilon z)=\delta_{i j}+o(\varepsilon|z|) \quad|g(\varepsilon z)|^{\frac{1}{2}}=1+o(\varepsilon|z|)
$$

We can define a map

$$
\begin{aligned}
\Phi_{\varepsilon} & : M \rightarrow \mathcal{N}_{\varepsilon} \\
\Phi_{\varepsilon}(\xi) & =t_{\varepsilon}\left(W_{\xi, \varepsilon}\right) W_{\xi, \varepsilon}
\end{aligned}
$$

Remark 8. We have that $W_{\varepsilon, \xi} \in H^{1}(M)$ and the following limits hold uniformly with respect to $\xi \in M$

$$
\begin{aligned}
\left\|W_{\varepsilon, \xi}\right\|_{\varepsilon} & \rightarrow\|U\|_{H^{1}\left(\mathbb{R}^{3}\right)} \\
\left|W_{\varepsilon, \xi}\right|_{\varepsilon, t} & \rightarrow\|U\|_{L^{t}\left(\mathbb{R}^{3}\right)} \text { for all } 2 \leq t \leq 6
\end{aligned}
$$

Lemma 9. We have that

$$
\lim _{\varepsilon \rightarrow 0} G_{\varepsilon}\left(W_{\varepsilon, \xi}\right)=G(U)=\int_{\mathbb{R}^{3}} q U^{2} \psi(U) d x
$$

uniformly with respect to $\xi \in M$

Proof. We set, for the sake of simplicity, $\psi_{\varepsilon}(x):=\psi_{\varepsilon}\left(W_{\varepsilon, \xi}\right)(x)$, and we define

$$
\tilde{\psi}_{\varepsilon}(z)=\psi_{\varepsilon}\left(\exp _{\xi}(\varepsilon z)\right) \chi_{r}(|\varepsilon z|) \text { for } z \in \mathbb{R}^{3}
$$

It is easy to see that $\left\|\tilde{\psi}_{\varepsilon}\right\|_{H^{1}\left(\mathbb{R}^{3}\right)} \leq C\left\|\psi_{\varepsilon}\right\|_{\varepsilon}$. Moreover, by (2)

$$
\left\|\psi_{\varepsilon}\right\|_{\varepsilon}^{2} \leq C\left\|W_{\varepsilon, \xi}\right\|_{\frac{12}{5}, \varepsilon}^{2}\left\|\psi_{\varepsilon}\right\|_{\varepsilon} \leq C\|U\|_{\frac{12}{5}}^{2}\left\|\psi_{\varepsilon}\right\|_{\varepsilon}
$$

so $\tilde{\psi}_{\varepsilon}$ is bounded in $H^{1}\left(\mathbb{R}^{3}\right)$ so there exists $\bar{\psi} \in H^{1}\left(\mathbb{R}^{3}\right)$ such that, up to extracting a subsequence, $\tilde{\psi}_{\varepsilon_{k}} \rightarrow \bar{\psi}$ weakly in $H^{1}\left(\mathbb{R}^{3}\right)$.

First, we want to prove that $\bar{\psi}$ is a weak solution of

$$
-\Delta v+v=q U^{2}
$$

that is $\bar{\psi}=\psi_{\infty}(U)$. Given $f \in C_{0}^{\infty}\left(\mathbb{R}^{3}\right)$, we have that the spt $f \subset B(0, T)$ for some $T>0$, so eventually spt $f \subset B\left(0, r / \varepsilon_{k}\right)$. Thus we can define

$$
f_{k}(x):=f\left(\frac{1}{\varepsilon_{k}} \exp _{\xi}^{-1}(x)\right)
$$

and we have that $f_{k}(x)$ is compactly supported in $B_{g}(\xi, r)$. By definition of $\psi_{\varepsilon}(x)$ we have that

$$
\int_{M} \varepsilon_{k}^{2} \nabla_{g} \psi_{\varepsilon_{k}} \nabla_{g} f_{k}+\psi_{\varepsilon_{k}} f_{k} d \mu_{g}=q \int_{M} W_{\varepsilon_{k}, \xi}^{2} f_{k} d \mu_{g}
$$


By the change of variables $x=\exp _{\xi}\left(\varepsilon_{k} z\right)$ and by (9) we get

$$
\begin{aligned}
\frac{1}{\varepsilon_{k}^{3}} \int_{M} \varepsilon_{k}^{2} \nabla_{g} \psi_{\varepsilon_{k}} \nabla_{g} f_{k}+\psi_{\varepsilon_{k}} f_{k} d \mu_{g} & =\int_{B\left(0, r / \varepsilon_{k}\right)}\left[g_{i j}\left(\varepsilon_{k} z\right) \partial_{i} \tilde{\psi}_{\varepsilon_{k}}(z) \partial_{j} f(z)+\tilde{\psi}_{\varepsilon_{k}}(z) f(z)\right]\left|g\left(\varepsilon_{k} z\right)\right|^{\frac{1}{2}} d z \\
& =\int_{B(0, T)} \nabla \tilde{\psi}_{\varepsilon_{k}}(z) \nabla f(z)+\tilde{\psi}_{\varepsilon_{k}}(z) f(z) d z+o\left(\varepsilon_{k}\right)
\end{aligned}
$$

thus, by weak convergence of $\tilde{\psi}_{\varepsilon}$ we get

$$
\frac{1}{\varepsilon_{k}^{3}} \int_{M} \varepsilon_{k}^{2} \nabla_{g} \psi_{\varepsilon_{k}} \nabla_{g} f_{k}+\psi_{\varepsilon_{k}} f_{k} d \mu_{g} \rightarrow \int_{\mathbb{R}^{3}} \nabla \bar{\psi}(z) \nabla f(z)+\bar{\psi}(z) f(z) d z .
$$

as $\varepsilon_{k} \rightarrow 0$. In the same way we get

$\frac{q}{\varepsilon_{k}^{3}} \int_{M} W_{\varepsilon_{k}, \xi}^{2} f_{k} d \mu_{g}=q \int_{B\left(0, r / \varepsilon_{k}\right)} U^{2}(z) f(z)\left|g\left(\varepsilon_{k} z\right)\right|^{\frac{1}{2}} d z=q \int_{\mathbb{R}^{3}} U^{2}(z) f(z) d z+o\left(\varepsilon_{k}\right)$

and

$$
\frac{q}{\varepsilon_{k}^{3}} \int_{M} W_{\varepsilon_{k}, \xi}^{2} f_{k} d \mu_{g} \rightarrow q \int_{\mathbb{R}^{3}} U^{2}(z) f(z) d z .
$$

By (10), (11), (12) we get that, for any $f \in C_{0}^{\infty}\left(\mathbb{R}^{3}\right)$ it holds

$$
\int_{\mathbb{R}^{3}} \nabla \bar{\psi} \nabla f+\bar{\psi} f=q \int_{\mathbb{R}^{3}} U^{2} f
$$

which proves that

$$
\tilde{\psi}_{\varepsilon_{k}} \rightarrow \psi_{\infty}(U) \text { weakly in } H^{1}\left(\mathbb{R}^{3}\right)
$$

To conclude, again by change of variables we have

$$
G_{\varepsilon_{k}}\left(W_{\varepsilon_{k}, \xi}\right)=\frac{1}{\varepsilon_{k}^{3}} \int_{B_{g}(\xi, r)} W_{\varepsilon_{k}, \xi}^{2} \psi\left(W_{\varepsilon_{k}, \xi}\right) d \mu_{g}=\int_{\mathbb{R}^{3}} U^{2}(z) \chi^{2}\left(\left|\varepsilon_{k} z\right|\right) \tilde{\psi}_{\varepsilon_{k}}\left|g\left(\varepsilon_{k} z\right)\right|^{\frac{1}{2}} d z .
$$

Since $U^{2} \in L^{6 / 5}\left(\mathbb{R}^{3}\right)$ one has

$$
U^{2}(z) \chi^{2}\left(\left|\varepsilon_{k} z\right|\right)\left|g\left(\varepsilon_{k} z\right)\right|^{\frac{1}{2}} \rightarrow U^{2}(z) \text { strongly in } L^{6 / 5}\left(\mathbb{R}^{3}\right),
$$

that, combined with (13) concludes the proof.

Proposition 10. For all $\varepsilon>0$ the map $\Phi_{\varepsilon}$ is continuous. Moreover for any $\delta>0$ there exists $\varepsilon_{0}=\varepsilon_{0}(\delta)$ such that, if $\varepsilon<\varepsilon_{0}$ then $I_{\varepsilon}\left(\Phi_{\varepsilon}(\xi)\right)<m_{\infty}+\delta$.

Proof. It is easy to see that $\Phi_{\varepsilon}$ is continuous because $t_{\varepsilon}(w)$ depends continuously on $w \in H_{g}^{1}(M)$.

At this point we prove that $t_{\varepsilon}\left(W_{\varepsilon, \xi}\right) \rightarrow 1$ uniformly with respect to $\xi \in M$. In fact, by Lemma $6 t_{\varepsilon}\left(W_{\varepsilon, \xi}\right)$ is the unique solution of

$$
t^{2}\left\|W_{\varepsilon, \xi}\right\|_{\varepsilon}^{2}+\omega G_{\varepsilon}\left(t W_{\varepsilon, \xi}\right)-t^{p}\left|W_{\varepsilon, \xi}\right|_{\varepsilon, p}^{p}=0
$$

which, in light of Remark 4 can by rewritten as

$$
\left\|W_{\varepsilon, \xi}\right\|_{\varepsilon}^{2}+\omega t^{2} G_{\varepsilon}\left(W_{\varepsilon, \xi}\right)-t^{p-2}\left|W_{\varepsilon, \xi}\right|_{\varepsilon, p}^{p}=0
$$

By Remark 8 and Lemma 9 we have the claim. In fact, we recall that, since $U$ is a solution of (7) it holds $\|U\|_{H^{1}\left(\mathbb{R}^{3}\right)}^{2}+\omega G(U)-|U|_{L^{p}\left(\mathbb{R}^{3}\right)}^{p}=0$.

At this point, we have

$$
I_{\varepsilon}\left(t_{\varepsilon}\left(W_{\varepsilon, \xi}\right) W_{\varepsilon, \xi}\right)=\left(\frac{1}{2}-\frac{1}{p}\right)\left\|W_{\varepsilon, \xi}\right\|_{\varepsilon}^{2} t_{\varepsilon}^{2}+\omega\left(\frac{1}{4}-\frac{1}{p}\right) t_{\varepsilon}^{4} G_{\varepsilon}\left(W_{\varepsilon, \xi}\right)
$$


Again, by Remark 8 and Lemma 9 and since $t_{\varepsilon}\left(W_{\varepsilon, \xi}\right) \rightarrow 1$ we have

$$
I_{\varepsilon}\left(t_{\varepsilon}\left(W_{\varepsilon, \xi}\right) W_{\varepsilon, \xi}\right) \rightarrow\left(\frac{1}{2}-\frac{1}{p}\right)\|U\|_{H^{1}\left(\mathbb{R}^{3}\right)}^{2}+\omega\left(\frac{1}{4}-\frac{1}{p}\right) G(U)=m_{\infty}
$$

that concludes the proof.

Remark 11. We set

$$
m_{\varepsilon}=\inf _{\mathcal{N}_{\varepsilon}} I_{\varepsilon}
$$

By Proposition 10 we have that

$$
\limsup _{\varepsilon \rightarrow 0} m_{\varepsilon} \leq m_{\infty}
$$

\section{THE MAP $\beta$}

For any $u \in \mathcal{N}_{\varepsilon}$ we can define a point $\beta(u) \in \mathbb{R}^{n}$ by

$$
\beta(u)=\frac{\int_{M} x \Gamma(u) d \mu_{g}}{\int_{M} \Gamma(u) d \mu_{g}}
$$

where $\Gamma(u)=\left(\frac{1}{2}-\frac{1}{p}\right) \frac{1}{\varepsilon^{3}}\left|u^{+}\right|^{p}-\frac{\omega}{4} \frac{1}{\varepsilon^{3}} u^{2} \psi_{\varepsilon}(u)$. Immediately one has that the function $\beta$ is well defined in $\mathcal{N}_{\varepsilon}$, since $\int_{M} \Gamma(u) d \mu_{g}=I_{\varepsilon}(u) \geq m_{\varepsilon}$

Lemma 12. There exists $\alpha>0$ such that $m_{\varepsilon} \geq \alpha$ for all $\varepsilon$.

Proof. Take $w$ such that $\left|w^{+}\right|_{\varepsilon, p}=1$, and $t_{\varepsilon}=t_{\varepsilon}(w)$ such that $t_{\varepsilon} w \in \mathcal{N}_{\varepsilon}$. By (6) we have

$$
I_{\varepsilon}\left(t_{\varepsilon} w\right)=\frac{t_{\varepsilon}^{2}}{4}\|w\|_{\varepsilon}^{2}+\left(\frac{1}{4}-\frac{1}{p}\right) t_{\varepsilon}^{p} \geq\left(\frac{1}{4}-\frac{1}{p}\right) t_{\varepsilon}^{p} .
$$

Moreover, we have that $\inf _{\left|w^{+}\right|_{\varepsilon, p}=1} t_{\varepsilon}(w)>0$. In fact, suppose that there exists a sequence $w_{n}$ such that $\left|w^{+}\right|_{\varepsilon, p}=1$ and $t_{\varepsilon}\left(w_{n}\right) \rightarrow 0$. Since $t_{\varepsilon}\left(w_{n}\right) w_{n} \in \mathcal{N}_{\varepsilon}$ it holds

$$
1=\left|w_{n}^{+}\right|_{\varepsilon, p}=\frac{1}{t_{\varepsilon}\left(w_{n}\right)^{p-2}}\left\|w_{n}\right\|_{\varepsilon}^{2}+\omega G_{\varepsilon}\left(t_{\varepsilon}\left(w_{n}\right)\right) \geq \frac{1}{t_{\varepsilon}\left(w_{n}\right)^{p-2}}\left\|w_{n}\right\|_{\varepsilon}^{2} .
$$

Also, we have that there exists a constant $C>0$ which does not depend on $\varepsilon$ such that $\left|w_{n}^{+}\right|_{\varepsilon, p} \leq\left|w_{n}\right|_{\varepsilon, p} \leq C\left\|w_{n}\right\|_{\varepsilon}$, so

$$
1 \geq \frac{1}{C t_{\varepsilon}\left(w_{n}\right)^{p-2}} \rightarrow+\infty
$$

that is a contradiction. This proves that $m_{\varepsilon} \geq \alpha$ for some $\alpha>0$.

Now we have to prove that, if $u \in \mathcal{N}_{\varepsilon} \cap I_{\varepsilon}^{m_{\infty}+\delta}$ then $\beta(u) \in M^{+}$.

Let us consider the following partitions of $M$. For a given $\varepsilon>0$ we say that a finite partition $\mathcal{P}_{\varepsilon}=\left\{P_{j}^{\varepsilon}\right\}_{j \in \Lambda_{\varepsilon}}$ of $M$ is a "good" partition if: for any $j \in \Lambda_{\varepsilon}$ the set $P_{j}^{\varepsilon}$ is closed; $P_{i}^{\varepsilon} \cap P_{j}^{\varepsilon} \subset \partial P_{i}^{\varepsilon} \cap \partial P_{j}^{\varepsilon}$ for any $i \neq j$; there exist $r_{1}(\varepsilon), r_{2}(\varepsilon)>0$ such that there are points $q_{j}^{\varepsilon} \in P_{j}^{\varepsilon}$ for which $B_{g}\left(q_{j}^{\varepsilon}, \varepsilon\right) \subset P_{j}^{\varepsilon} \subset B_{g}\left(q_{j}^{\varepsilon}, r_{2}(\varepsilon)\right) \subset B_{g}\left(q_{j}^{\varepsilon}, r_{1}(\varepsilon)\right)$, with $r_{1}(\varepsilon) \geq r_{2}(\varepsilon) \geq C \varepsilon$ for some positive constant $C$; lastly, there exists a finite number $\nu(M) \in \mathbb{N}$ such that every $\xi \in M$ is contained in at most $\nu(M)$ balls $B_{g}\left(q_{j}^{\varepsilon}, r_{1}(\varepsilon)\right)$, where $\nu(M)$ does not depends on $\varepsilon$. 
Remark 13. We recall that there exists a constant $\gamma>0$ such that, for any $\delta>0$ and for any $\varepsilon<\varepsilon_{0}(\delta)$ as in Proposition 10, given any "good" partition $\mathcal{P}_{\varepsilon}=\left\{P_{j}^{\varepsilon}\right\}_{j}$ of the manifold $M$ and for any function $u \in \mathcal{N}_{\varepsilon} \cap I_{\varepsilon}^{m_{\infty}+\delta}$ there exists, for an index $\bar{j}$ a set $P_{\bar{j}}^{\varepsilon}$ such that

$$
\frac{1}{\varepsilon^{3}} \int_{P_{\bar{j}}^{\varepsilon}}\left|u^{+}\right|^{p} d x \geq \gamma
$$

Indeed we can proceed verbatim as in Lemma 12 of [11], considering that, since $I^{\prime}(u)[u]=0$,

$$
\begin{aligned}
\|u\|_{\varepsilon}^{2} & =\left|u^{+}\right|_{\varepsilon, p}^{p}-\frac{1}{\varepsilon^{3}} \int_{M} \omega u^{2} \psi(u) \leq\left|u^{+}\right|_{\varepsilon, p}^{p} \\
& =\sum_{j}\left|u_{j}^{+}\right|_{\varepsilon, p}^{p} \leq \max _{j}\left\{\left|u_{j}^{+}\right|_{\varepsilon, p}^{p-2}\right\} \sum_{j}\left|u_{j}^{+}\right|_{\varepsilon, p}^{2}
\end{aligned}
$$

where $u_{j}^{+}$is the restriction of the function $u^{+}$on the set $P_{j}$, and arguing as in Lemma 5.3 of [3], we obtain that for some $C>0$ it holds $\sum_{j}\left|u_{j}^{+}\right|_{\varepsilon, p}^{2} \leq C \nu\left\|u^{+}\right\|_{\varepsilon}^{2}$, and there the proof follows since

$$
\max _{j}\left\{\left|u_{j}^{+}\right|_{\varepsilon, p}^{p-2}\right\} \geq \frac{1}{C \nu} .
$$

Proposition 14. For any $\eta \in(0,1)$ there exists $\delta_{0}<m_{\infty}$ such that for any $\delta \in$ $\left(0, \delta_{0}\right)$ and any $\varepsilon \in\left(0, \varepsilon_{0}(\delta)\right)$ as in Proposition 10 , for any function $u \in \mathcal{N}_{\varepsilon} \cap I_{\varepsilon}^{m_{\infty}+\delta}$ we can find a point $q=q(u) \in M$ such that

$$
\int_{B_{g}(q, r / 2)} \Gamma(u)>(1-\eta) m_{\infty} .
$$

Proof. First, we prove the proposition for $u \in \mathcal{N}_{\varepsilon} \cap I_{\varepsilon}^{m_{\varepsilon}+2 \delta}$.

By contradiction, we assume that there exists $\eta \in(0,1)$ such that we can find two sequences of vanishing real number $\delta_{k}$ and $\varepsilon_{k}$ and a sequence of functions $\left\{u_{k}\right\}_{k}$ such that $u_{k} \in \mathcal{N}_{\varepsilon_{k}}$,

$m_{\varepsilon_{k}} \leq I_{\varepsilon_{k}}\left(u_{k}\right)=\left(\frac{1}{2}-\frac{1}{p}\right)\left\|u_{k}\right\|_{\varepsilon_{k}}^{2}+\omega\left(\frac{1}{4}-\frac{1}{p}\right) G_{\varepsilon_{k}}\left(u_{k}\right) \leq m_{\varepsilon_{k}}+2 \delta_{k} \leq m_{\infty}+3 \delta_{k}$ for $k$ large enough (see Remark 11), and, for any $q \in M$,

$$
\int_{B_{g}(q, r / 2)} \Gamma\left(u_{k}\right) \leq(1-\eta) m_{\infty}
$$

By Ekeland principle and by definition of $\mathcal{N}_{\varepsilon_{k}}$ we can assume

$$
\left|I_{\varepsilon_{k}}^{\prime}\left(u_{k}\right)[\varphi]\right| \leq \sigma_{k}\|\varphi\|_{\varepsilon_{k}} \text { where } \sigma_{k} \rightarrow 0 .
$$

By Remark 13 there exists a set $P_{k}^{\varepsilon_{k}} \in \mathcal{P}_{\varepsilon_{k}}$ such that

$$
\frac{1}{\varepsilon_{k}^{3}} \int_{P_{k}^{\varepsilon_{k}}}\left|u_{k}^{+}\right|^{p} d \mu_{g} \geq \gamma
$$

so, we choose a point $q_{k} \in P_{k}^{\varepsilon_{k}}$ and we define, in analogy with the proof of Lemma 9

$$
w_{k}(z):=u_{k}\left(\exp _{q_{k}}\left(\varepsilon_{k} z\right)\right) \chi\left(\varepsilon_{k}|z|\right)
$$


where $z \in B\left(0, r / \varepsilon_{k}\right) \subset \mathbb{R}^{3}$. Extending trivially $w_{k}$ by zero to the whole $\mathbb{R}^{3}$ we have that $w_{k} \in H^{1}\left(\mathbb{R}^{3}\right)$ and, by (16),

$$
\left\|w_{k}\right\|_{H^{1}\left(\mathbb{R}^{3}\right)}^{2} \leq C\left\|u_{k}\right\|_{\varepsilon_{k}}^{2} \leq C .
$$

So there exists a $w \in H^{1}\left(\mathbb{R}^{3}\right)$ such that, up to subsequences, $w_{k} \rightarrow w$ weakly in $H^{1}\left(\mathbb{R}^{3}\right)$ and strongly in $L_{\text {loc }}^{t}\left(\mathbb{R}^{3}\right)$ for $2 \leq t<6$. Moreover we set $\psi_{k}(x):=\psi_{\varepsilon}\left(u_{k}\right)(x)$ and $\tilde{\psi}_{k}=\psi_{k}\left(\exp _{q_{k}}\left(\varepsilon_{k} z\right)\right) \chi\left(\varepsilon_{k}|z|\right)$. Arguing as in Lemma 9 we get that $\tilde{\psi}_{k} \rightarrow \psi_{\infty}(w)$ weakly in $H^{1}\left(\mathbb{R}^{3}\right)$ and strongly in $L_{\text {loc }}^{t}\left(\mathbb{R}^{3}\right)$ for all $2 \leq t<6$.

Again, given $f \in C_{0}^{\infty}\left(\mathbb{R}^{3}\right)$, with spt $f \subset B(0, T)$ for some $T>0$ we can define

$$
f_{k}(x):=f\left(\frac{1}{\varepsilon_{k}} \exp _{\xi}^{-1}(x)\right)
$$

and, by (17) we have $\left|I_{\varepsilon_{k}}^{\prime}\left(u_{k}\right)\left[f_{k}\right]\right| \rightarrow 0$ as $k \rightarrow \infty$. Now, by change of variables we have

$$
\begin{aligned}
I_{\varepsilon_{k}}^{\prime}\left(u_{k}\right)\left[f_{k}\right] & =\frac{1}{\varepsilon_{k}^{3}} \int_{M} \varepsilon_{k}^{2} \nabla_{g} u_{k} \nabla_{g} f_{k}+u_{k} f_{k}+\omega q u_{k} \psi_{k} f_{k}-\left(u_{k}^{+}\right)^{p-1} f_{k} d \mu_{g} \\
& =\int_{B(0, T)}\left[g_{i j}\left(\varepsilon_{k}\right) \partial_{i} w_{k} \partial_{j} f+w_{k} f+\omega q w_{k} \tilde{\psi}_{k} f-\left(w_{k}^{+}\right)^{p-1} f\right]\left|g\left(\varepsilon_{k} z\right)\right|^{\frac{1}{2}} d z \\
& =\int_{\mathbb{R}^{3}} \nabla w_{k} \nabla f+w_{k} f+\omega q w_{k} \tilde{\psi}_{k} f-\left(w_{k}^{+}\right)^{p-1} f d z+o\left(\varepsilon_{k}\right) \\
& \rightarrow \int_{\mathbb{R}^{3}} \nabla w \nabla f+w f+\omega q w \psi_{\infty}(w) f-\left(w_{k}^{+}\right)^{p-1} f d z=I_{\infty}^{\prime}(w)[f]
\end{aligned}
$$

and, by (17), we get that $w$ is a weak solution of the limit problem (7) and that $w \in \mathcal{N}_{\infty}$. By Lemma 13 and by the choice of $q_{k}$ we have that $w \neq 0$, so $w>0$ and $I_{\infty}(w) \geq m_{\infty}$.

Now, consider the functions

$$
h_{k}:=\frac{1}{\varepsilon^{3}}\left|u_{k}^{+}\right|^{\frac{1}{p}}\left(\exp _{q_{k}}\left(\varepsilon_{k} z\right)\right) \mid g_{q_{k}}\left(\varepsilon_{k} z\right)^{\frac{1}{2 p}} \mathbb{I}_{B_{g}\left(q_{k}, r\right)}
$$

where $\mathbb{I}_{B_{g}\left(q_{k}, r\right)}$ is the indicatrix function on $B_{g}\left(q_{k}, r\right)$. Since $\left|u_{k}\right|_{\varepsilon, p}$ is bounded, then $h_{k}$ is bounded in $L^{p}\left(\mathbb{R}^{3}\right)$ so, it converges weakly to some $\bar{h} \in L^{p}\left(\mathbb{R}^{3}\right)$. We have that $h=\left|w^{+}\right|^{\frac{1}{p}}$. Take $f \in C_{0}^{\infty}\left(\mathbb{R}^{3}\right)$, with spt $f \subset B(0, T)$ for some $T>0$. Since, eventually $B(0, T) \subset B\left(0, r / 2 \varepsilon_{k}\right),\left|u_{k}^{+}\right|^{\frac{1}{p}}\left(\exp _{q_{k}}\left(\varepsilon_{k} z\right)\right)=w_{k}^{+}$on $B(0, T)$. Moreover, on $B(0, T)$ we have that $\left|g_{q_{k}}\left(\varepsilon_{k} z\right)\right|^{\frac{1}{2 p}}=1+o\left(\varepsilon_{k}\right)$. Thus, since $w_{k} \rightarrow w$ in $L^{p}\left(\mathbb{R}^{3}\right)$ we get.

$$
\int_{\mathbb{R}^{3}} h_{k} f d z \rightarrow \int_{\mathbb{R}^{3}}\left|w^{+}\right|^{\frac{1}{p}} f d z
$$

for any $f \in C_{0}^{\infty}\left(\mathbb{R}^{3}\right)$. In the same way we can consider the functions

$$
\begin{gathered}
j_{k}=\frac{1}{\varepsilon^{3}}\left(g_{i j}\left(\varepsilon_{k} z\right) \partial_{i} u_{k}\left(\exp _{q_{k}}\left(\varepsilon_{k} z\right)\right) \partial_{j} u_{k}\left(\exp _{q_{k}}\left(\varepsilon_{k} z\right)\right)\left|g_{q_{k}}\left(\varepsilon_{k} z\right)\right|^{\frac{1}{2}}\right)^{\frac{1}{2}} \mathbb{I}_{B_{g}\left(q_{k}, r\right)} \\
l_{k}:=\frac{1}{\varepsilon^{3}}\left|u_{k}\right|^{\frac{1}{2}}\left(\exp _{q_{k}}\left(\varepsilon_{k} z\right)\right)\left|g_{q_{k}}\left(\varepsilon_{k} z\right)\right|^{\frac{1}{4}} \mathbb{I}_{B_{g}\left(q_{k}, r\right)}
\end{gathered}
$$

We have that $j_{k}, l_{k} \in L^{2}\left(\mathbb{R}^{3}\right)$ and that $j_{k} \rightarrow|\nabla w|^{\frac{1}{2}}, l_{k} \rightarrow|w|^{\frac{1}{2}}$ in $L^{2}\left(\mathbb{R}^{3}\right)$. Thus we have 
At this point, since $w \in \mathcal{N}_{\infty}$ and by (16) we get

$$
\begin{aligned}
m_{\infty} & \leq I_{\infty}(w)=\frac{1}{4}\|w\|_{H^{1}}^{2}+\left(\frac{1}{4}-\frac{1}{p}\right)\left|w^{+}\right|_{p}^{p} \\
& \leq \liminf _{k \rightarrow \infty} \frac{1}{4}\left\|j_{k}\right\|_{L^{2}}^{2}+\frac{1}{4}\left\|i_{k}\right\|_{L^{2}}^{2}+\left(\frac{1}{4}-\frac{1}{p}\right)\left|h_{k}\right|_{p}^{p} \\
& \leq \frac{1}{4}\left\|u_{k}\right\|_{\varepsilon}^{2}+\left(\frac{1}{4}-\frac{1}{p}\right)\left|u_{k}^{+}\right|_{p}^{p} \leq m_{\infty}+3 \delta_{k}
\end{aligned}
$$

so we have that $w$ is a ground state for the limit problem (7).

Given $T>0$, by the definition of $w_{k}$ we get, for $k$ large enough

$$
\begin{gathered}
\int_{B(0, T)}\left[\left(\frac{1}{2}-\frac{1}{p}\right)\left(w_{k}^{+}\right)^{p}-\frac{\omega}{4} w_{k}^{2} \tilde{\psi}_{k}\right] g\left(\varepsilon_{k} z\right) d z \\
=\frac{1}{\varepsilon^{3}} \int_{B\left(q_{k}, \varepsilon_{k} T\right)}\left(\frac{1}{2}-\frac{1}{p}\right)\left(u_{k}^{+}\right)^{p}-\frac{\omega}{4} u_{k}^{2} \psi_{\varepsilon}\left(u_{k}\right) d \mu_{g} \\
\quad=\int_{B\left(q_{k}, \varepsilon_{k} T\right)} \Gamma\left(u_{k}\right) d x \leq \int_{B\left(q_{k}, r / 2\right)} \Gamma\left(u_{k}\right) d x \leq(1-\eta) m_{\infty}
\end{gathered}
$$

and, if we choose $T$ sufficiently big, this leads to a contradiction since $w_{k} \rightarrow w$ and $\tilde{\psi}_{k} \rightarrow \psi_{\infty}(w)$ in $L^{t}(B(0, T))$ for any $T>0$. Since $m_{\infty}=I_{\infty}(w)=\left(\frac{1}{2}-\frac{1}{p}\right)\left|w^{+}\right|^{p}-$ $\frac{\omega}{4} G(w)$, it is possible to choose $T$ such that (18) is false, so the lemma is proved for $u \in \mathcal{N}_{\varepsilon} \cap I_{\varepsilon}^{m_{\varepsilon}+2 \delta}$.

The above arguments also prove that

$$
\liminf _{k \rightarrow \infty} m_{\varepsilon_{k}} \geq \lim _{k \rightarrow \infty} I_{\varepsilon_{k}}\left(u_{k}\right)=m_{\infty} .
$$

and, in light of (14), this leads to

$$
\lim _{\varepsilon \rightarrow 0} m_{\varepsilon}=m_{\infty} .
$$

Hence, when $\varepsilon, \delta$ are small enough, $\mathcal{N}_{\varepsilon} \cap I_{\varepsilon}^{m_{\infty}+\delta} \subset \mathcal{N}_{\varepsilon} \cap I_{\varepsilon}^{m_{\varepsilon}+2 \delta}$ and the general claim follows.

Proposition 15. There exists $\delta_{0} \in\left(0, m_{\infty}\right)$ such that for any $\delta \in\left(0, \delta_{0}\right)$ and any $\varepsilon \in\left(0, \varepsilon\left(\delta_{0}\right)\right.$ (see Proposition [10), for every function $u \in \mathcal{N}_{\varepsilon} \cap I_{\varepsilon}^{m_{\infty}+\delta}$ it holds $\beta(u) \in M^{+}$. Moreover the composition

$$
\beta \circ \Phi_{\varepsilon}: M \rightarrow M^{+}
$$

is $s$ homotopic to the immersion $i: M \rightarrow M^{+}$

Proof. By Proposition 14, for any function $u \in \mathcal{N}_{\varepsilon} \cap I_{\varepsilon}^{m_{\infty}+\delta}$, for any $\eta \in(0,1)$ and for $\varepsilon, \delta$ small enough, we can find a point $q=q(u) \in M$ such that

$$
\int_{B(q, r / 2)} \Gamma(u)>(1-\eta) m_{\infty}
$$

Moreover, since $u \in \mathcal{N}_{\varepsilon} \cap I_{\varepsilon}^{m_{\infty}+\delta}$ we have

$$
I_{\varepsilon}(u)=\int_{M} \Gamma(u) \leq m_{\infty}+\delta .
$$


Hence

$$
\begin{aligned}
|\beta(u)-q| & \leq \frac{\left|\int_{M}(x-q) \Gamma(u)\right|}{\int_{M} \Gamma(u)} \\
& \leq \frac{\left|\frac{1}{\varepsilon^{3}} \int_{B(q, r / 2)}(x-q) \Gamma(u)\right|}{\int_{M} \Gamma(u)}+\frac{\left|\frac{1}{\varepsilon^{3}} \int_{M \backslash B(q, r / 2)}(x-q) \Gamma(u)\right|}{\int_{M} \Gamma(u)} \\
& \leq \frac{r}{2}+2 \operatorname{diam}(M)\left(1-\frac{1-\eta}{1+\delta / m_{\infty}}\right),
\end{aligned}
$$

and the second term can be made arbitrarily small, choosing $\eta, \delta$ and $\varepsilon$ sufficiently small. The second claim of the theorem is standard.

Acknowledgments. Every problem has a story and an inspiration. This one comes out from an interesting remark of Professor Ireneo Peral during a conference in Alghero (Italy). We hence would like to thank Prof. Peral for his keen and inspiring suggestion.

\section{REFERENCES}

[1] A. Ambrosetti, D. Ruiz, Multiple bound states for the Schroedinger-Poisson problem, Commun. Contemp. Math. 10 (2008) 391-404

[2] A. Azzollini, A. Pomponio, Ground state solutions for the nonlinear Schroedinger-Maxwell equations, J. Math. Anal. Appl. 345 (2008) no. 1, 90-108

[3] V. Benci, C. Bonanno, A.M. Micheletti, On the multiplicity of solutions of a nonlinear elliptic problem on Riemannian manifolds, Journal of Functional Analysis 252 (2007) 464-489.

[4] V. Benci, G. Cerami, The effect of the domain topology on the number of positive solutions of nonlinear elliptic problems, Arch. Ration. Mech. Anal. 114 (1991) 79-93.

[5] V.Benci, D.Fortunato, An eigenvalue problem for the Schroedinger-Maxwell equations, Topol. Methods Nonlinear Anal. 11 (1998), no. 2, 283- 293.

[6] G.M.Coclite, A multiplicity result for the nonlinear Schroedinger-Maxwell equations, Commun. Appl. Anal. 7 (2003), 417-423.

[7] T. D'Aprile, D. Mugnai, Solitary waves for nonlinear Klein-Gordon-Maxwell and Schroedinger-Maxwell equations, Proc. Roy. Soc. Edinburgh Sect. A 134 (2004), no. 5, 893-906.

[8] P. D'Avenia, Non-radially symmetric solutions of nonlinear Schroedinger equation coupled with Maxwell equations, Adv. Nonl. Studies 2 (2002), 177-192

[9] P. D’Avenia, M. Squassina, Soliton dynamics for the Schroedinger-Newton system, Math. Models Methods Appl. Sci. 24 (2014), 553-572.

[10] M. Ghimenti, A.M. Micheletti, Number and profile of low energy solutions for singularly perturbed Klein Gordon Maxwell systems on a Riemannian manifold, J. Differential Equations 256 (2014), no. 7, 2502-2525.

[11] M. Ghimenti, A.M. Micheletti, Low energy solutions for the semiclassical limit of Schroedinger-Maxwell systems, Progress in Nonlinear Differential Equations and their Applications 85 (2014), no. 7, 287-300.

[12] X. He, Multiplicity and concentration of positive solutions for the Schroedinger-Poisson equations, Z. Angew. Math. Phys. 62 (2011), 869-889.

[13] I.Ianni Sign-changing radial solutions for the Schroedinger-Poisson-Slater problem Topol. Meth. Nonlin. An. 41 (2013), 365-385

[14] I.Ianni. D.Ruiz Ground and bound states for a static Schroedinger-Poisson-Slater problem Comm. Cont. Math. 14 (2012), 22p.

[15] Kikuchi, On the existence of solutions for a elliptic system related to the MaxwellSchroedinger equations, Non- linear Anal. 67 (2007) 1445-1456.

[16] D. Ruiz, The Schroedinger-Poisson equation under the effect of a nonlinear local term, Journ. of Functional Analysis 237 (2006), 655-674.

[17] D. Ruiz, On the Schroedinger-Poisson-Slater System: Behavior of Minimizers, Radial and Nonradial Cases, Arch. Rat. Mech. An. 198 (2010), 349-368. 
M. Ghimenti,

Dipartimento di Matematica Università di Pisa Largo B. Pontecorvo 5, 56100 Pisa, ITALY

E-mail address: ghimenti@mail.dm.unipi.it

A. M. Micheletti,

Dipartimento di Matematica Università di Pisa Largo B. Pontecorvo 5, 56100 Pisa, ITALY

E-mail address: a.micheletti@dma.unipi.it. 